\title{
A Clinical Study on Extrapulmonary Tuberculosis
}

\author{
MM KARIM $^{\mathrm{a}}$, SA CHOWDHURY ${ }^{\mathrm{b}}$, MM HUSSAIN $^{\mathrm{c}}$, MA FAIZ $^{\mathrm{d}}$
}

\begin{abstract}
Summary:
Findings of 80 patients of extra-pulmonary tuberculosis are described in this study. Most of the patients were under 30 years of age (71.2\%), female patients were $56.3 \%$ and housewives were $37.3 \%$. Lower socio-economic class were commonly affected (66.2\%). Eighteen patients (22.5\%) were smoker and almost equal number of cases had the history of intake of un-boiled milk. $44 \%$ patients were not vaccinated against tuberculosis. $36.2 \%$ patients had history of contact with tuberculous patients and $18.8 \%$ had previous history of tuberculosis. $70 \%$ patients had the history of fever and $30 \%$
\end{abstract}

\section{Introduction:}

Tuberculosis (TB) can affect almost any organ of the body and although the most common presentation is pulmonary, extra pulmonary disease is not rare. The sites involved in extra-pulmonary tuberculosis are lymph nodes, abdomen, bones, joints including spine, genitourinary system and central nervous system. Some patients may present with miliary tuberculosis. Others may present with abscess, fistula and cutaneous lesion. Presentation of extra-pulmonary disease may be atypical or relatively insidious and tuberculosis may not be considered initially in differential diagnosis. This is an important phenomenon as delay in diagnosis may be crippling or even life threatening.

In a survey of notification of Tuberculosis in England and Wales conducted by Medical Research Council (MRC) in $1983^{1}$ the disease was classified as pulmonary in $68 \%$, extra-pulmonary $25 \%$ and both $7 \%$. Hence, $32 \%$ of newly notified patients with tuberculosis presented with extra pulmonary manifestation. For the patients of Indian Subcontinent (ISC) in the survey the figure were $54 \%, 34 \%$ and $12 \%$ respectively. Hence, $46 \%$ of newly diagnosed patients with tuberculosis in ISC had extra pulmonary tuberculosis. In 1983 the overall notification

a. Dr. Mohammad Masud Karim, Junior Consultant of Surgery, Chittagong Medical College Hospital, Chittagong.

b. Dr Safiul Azam Chowdhury, FCPS(Surgery) Part-II student.

c. Professor Md. Margub Hussain, Professor of Surgery, Chittagong Medical College.

d. Professor M A Faiz, Professor of Medicine, Dhaka Medical College.

Address of correspondence: Dr. Mohammad Masud Karim, Junior Consultant of Surgery, Ward No-25, Chittagong Medical College Hospital, Chittagong. Email: drmmkarim@yahoo.com had history of cough. Significant weight loss was noted in $85 \%$ patients. Lymph node tuberculosis was $36.2 \%$, abdominal tuberculosis 35\%. Cervical lymph nodes alone (37.9\%) were commonly affected among the lymph node tuberculosis. Diagnosis was mainly based on histopathological examination or biopsy of specimen (97.5\%) and demonstrations of AFB was possible in $2.5 \%$ cases. Along with surgical treatment medical treatment (chemotherapy) were prescribed in every patient. Forty-eight patients came for follow up. All responded to anti tubercular chemotherapy.

(J Bangladesh Coll Phys Surg 2006; 24: 19-28)

rate for non-respiratory disease was 51 times as high for the ISC (81per 100000) as for the white group (1.6 per 100000). In a more recent survey of 2163 previously untreated patients notified in $1988,698(32 \%)$ had extrapulmonary tuberculosis of which 395 (57\%) were of ISC ethnic origin. ${ }^{2}$

In the USA the proportion of all reported cases of extra pulmonary tuberculosis has risen from $8 \%$ in 1964 to $15 \%$ in 1981 and $17.5 \%$ in $1986^{3,4}$. The same trends have been observed in Hong Kong.

The patients infected with HIV have a high risk of developing tuberculosis of all forms particularly extrapulmonary ones like lymph node and meningeal TB. TB is also rapidly progressive in HIV infected person. ${ }^{5}$

Hence the recognition and diagnosis of extra pulmonary lesion are likely to assume a greater importance in the foreseeable future.

The objective of the present study was to identify the various presentation of extra pulmonary tuberculosis in our country, to see the response to $1^{\text {st }}$ line anti tuberculous treatment and also to evaluate problems related to patient management. This may form the basis of further study and might help in planning and policy making in future.

Materials and methods:

This was a prospective, cross-sectional, observational study conducted among the patients who were admitted either in Chittagong Medical College Hospital or other city hospitals in Chittagong with features of extra pulmonary tuberculosis. The study period was August 2001 to May 2003. A detailed history, complete physical examination, various 
laboratory work and radiological studies were carried out. Histological confirmation was tried in every case. Diagnosis of TB was made by histological or cytological examination or demonstration of Acid Fast Bacillus. Informed consent was taken from the patient or guardian about participation in the study with the right not to participate.

The cases were treated with anti tuberculosis chemotherapy (WHO Schedule) and the patients were followed up in a follow up clinic during the period of chemotherapy.

All findings were noted in case record form. The results were calculated and interpreted through appropriate statistical analysis with the help of a statistician and presented in tables and other illustrations.

\section{Results:}

During the study period 80 patients of extra pulmonary tuberculosis were diagnosed and treated. Of them sixteen were transferred from other disciplines for surgical intervention. All patients received anti tuberculous chemotherapy according to the recommendation of WHO and they were followed up in a follow up clinic. Adjustment of drug was done or changed depending on the response and complications.

Among the patients 35 were male and 45 were female. Female patients predominated in this series. Age ranged between 13 to 62 years with a mean age of $27.5 \pm 11.1$. Clinical presentation was variable. Most common manifestation was neck swelling due to tuberculous cervical lymphadenopathy. Abdominal pain and distension was present in 18 and 14 cases respectively. Classical presentation of lump in the right iliac fossa was present in ten patients. One patient presented with ulceration near the anal canal without any internal pathology. One patient had carcinoma stomach and peritoneal tuberculosis. The mode of presentation is shown in Table-I.

Table-I

Mode of presentation of cases of extra-pulmonary tuberculosis $(n=80)$.

\begin{tabular}{|c|c|c|c|}
\hline \multicolumn{2}{|l|}{ Presentation } & Number of cases & Percentage \\
\hline \multicolumn{2}{|l|}{ Neck Swelling } & 25 & 31.2 \\
\hline \multicolumn{2}{|l|}{ Abdominal pain } & 18 & 22.5 \\
\hline \multicolumn{2}{|l|}{ Abdominal distension } & 14 & 17.5 \\
\hline \multicolumn{2}{|c|}{ Swelling over different parts of the body } & 9 & 11.2 \\
\hline \multicolumn{2}{|c|}{ Abdominal lump, right iliac fossa } & 8 & 10.0 \\
\hline \multicolumn{2}{|c|}{ Fever } & 7 & 8.7 \\
\hline \multicolumn{2}{|l|}{ Vomiting } & 6 & 7.5 \\
\hline \multicolumn{2}{|l|}{ Swelling in the axilla } & 6 & 7.5 \\
\hline \multirow[t]{2}{*}{ Lump in the breasts } & Right & 3 & 6.2 \\
\hline & Left & 2 & \\
\hline \multicolumn{2}{|l|}{ Swelling over sternum } & 2 & 2.5 \\
\hline \multicolumn{2}{|l|}{ Scrotal swelling } & 2 & 2.5 \\
\hline \multicolumn{2}{|l|}{ Per rectal bleeding } & 1 & 1.2 \\
\hline \multicolumn{2}{|l|}{ Jaw swelling } & 1 & 1.2 \\
\hline \multicolumn{2}{|c|}{ Discharging sinus over back } & 1 & 1.2 \\
\hline \multicolumn{2}{|c|}{ Swelling in the right groin } & 1 & 1.2 \\
\hline \multicolumn{2}{|c|}{ Perianal swelling } & 1 & 1.2 \\
\hline \multicolumn{2}{|c|}{ Swelling in the laparotomy wound } & 1 & 1.2 \\
\hline \multicolumn{2}{|c|}{ Low back pain } & 1 & 1.2 \\
\hline \multicolumn{2}{|c|}{ Inability to move limbs } & 1 & 1.2 \\
\hline \multicolumn{2}{|l|}{ Constipation } & 1 & 1.2 \\
\hline \multicolumn{2}{|c|}{ Swelling in the right iliac region } & 1 & 1.2 \\
\hline \multicolumn{2}{|c|}{ Multiple discharging sinus of left foot } & 1 & 1.2 \\
\hline \multicolumn{2}{|c|}{ Discharging sinus over sternum } & 1 & 1.2 \\
\hline \multicolumn{2}{|c|}{ Discharging sinus from appendicectomy wound } & 1 & 1.2 \\
\hline \multicolumn{2}{|c|}{ Perianal ulcer } & 1 & 1.2 \\
\hline
\end{tabular}


Young patients of 12 to 30 years accounted for about three fourth of total cases. Age distribution of this series is shown in Table-II.

Table-III shows relationship of age and tuberculosis of different extra-pulmonary site. Both cervical lymphadenopathy and abdominal tuberculosis occured at similar age. Comparatively late presentation was seen in bone (spinal) and musculoskeletal tuberculosis.

Relationship between different occupation and tuberculosis at different sites were studied. Housewives residing at home by far the largest group
(37.5\%). Young students were also common victims (17.5\%).

People of lower income group were found to be more commonly affected confirming the higher prevalence in overcrowded, unhygienic living condition and having probable malnutrition. $66.2 \%$ TB were seen in lower class whereas in higher class it was only $6.2 \%$.

Eighteen $(22.5 \%)$ out of 80 patients were smoker while $17(21.2 \%)$ had history of taking un-boiled milk.

Table-II

\section{Distribution of age and sex among the different varieties of extra-pulmonary tuberculosis $(n=80)$}

\begin{tabular}{|c|c|c|c|c|c|c|c|c|c|c|c|}
\hline \multirow{2}{*}{\multicolumn{2}{|c|}{ Types of EPT* }} & \multicolumn{6}{|c|}{ Age Groups } & \multicolumn{2}{|c|}{ Sex } & \multirow[t]{2}{*}{ Total } & \multirow[t]{2}{*}{$\%$} \\
\hline & & $12-20$ & $21-30$ & $31-40$ & $41-50$ & $51-60$ & $61-70$ & Male & Female & & \\
\hline \multicolumn{2}{|c|}{ Lymph Node TB } & 11 & 10 & 4 & 4 & & & 10 & 19 & 29 & 36.2 \\
\hline \multicolumn{2}{|c|}{ Abdominal TB } & 8 & 9 & 7 & 3 & & 1 & 13 & 15 & 28 & 35 \\
\hline \multicolumn{2}{|c|}{ Taberculous Abscess } & 2 & 6 & 1 & & & & 7 & 2 & 9 & 11.2 \\
\hline \multirow[t]{2}{*}{ Breast TB } & Right & & 3 & & & & & & 3 & 5 & 6.2 \\
\hline & Left & & 1 & & & 1 & & & 2 & & \\
\hline \multicolumn{2}{|c|}{ Musculo-skeletalTB } & 1 & 1 & 1 & & & & 1 & 2 & 3 & 3.7 \\
\hline \multicolumn{2}{|l|}{ Spine TB } & 1 & & & & 1 & & & 2 & 2 & 2.5 \\
\hline \multicolumn{2}{|c|}{ Perianal abscess, ulcer } & 1 & 1 & & & & & 2 & & 2 & 2.5 \\
\hline \multicolumn{2}{|c|}{ Genital TB (Testis) } & 1 & 1 & & & & & 2 & & 2 & 2.5 \\
\hline \multicolumn{2}{|l|}{ Total } & 25 & 32 & 13 & 7 & 2 & 1 & 35 & 45 & 80 & \\
\hline \multicolumn{2}{|l|}{ Percentage } & 31.2 & 40 & 16.25 & 8.7 & 2.5 & 1.2 & 43.7 & 56.3 & & \\
\hline
\end{tabular}

*EPT: Extra-pulmonary tuberculosis.

Table-III

Age distribution in different categories of extra-pulmonary tuberculosis $(n=80)$.

\begin{tabular}{lcccccc} 
TB Type & Number & Minimum & Maximum & Mean & SD & $\begin{array}{c}\text { \% of Total } \\
(\mathrm{n}=80)\end{array}$ \\
\hline Lymph node TB & 29 & 13 & 50 & 26.2 & 10.4 & 36.2 \\
Abdominal TB & 28 & 13 & 62 & 28.2 & 11.4 & 35 \\
Tuberculous abscess & 9 & 13 & 45 & 27 & 9.2 & 11.2 \\
Breast TB & 5 & 20 & 60 & 31.2 & 16.3 & 6.2 \\
Musculoskeletal TB & 3 & 22 & 40 & 28 & 10.4 & 3.7 \\
Spine TB & 2 & 15 & 55 & 35 & 28.3 & 2.5 \\
Perianal abscess \& ulcer & 2 & 27 & 27 & 27 & 27.2 & 2.5 \\
TB testes & 2 & 20 & 20 & 20 & 18.3 & 2.5 \\
All cases & 75 & 13 & 62 & 27.5 & 11.1 & 100 \\
\hline
\end{tabular}


$56 \%$ patients were vaccinated against tuberculosis and $44 \%$ were not. Scar marks of vaccine were present in $97.6 \%$ of patients who were vaccinated.

$18.8 \%(\mathrm{n}=15)$ patients had previous history of tuberculosis, among which 11 patients $(73.3 \%)$ were treated incompletely. 36.2\% $(n=29)$ patients had history of contact with a patient of pulmonary tuberculosis. $23.8 \%(\mathrm{n}=19)$ patients had definite family history of tuberculosis where most of the family member $(n=13)$ were treated and declared cured.

Out of 80 patients in this series $42(52.5 \%)$ were living in over crowded rooms. One patient had diabetes mellitus and one was on steroid therapy. Both contributed to resistance.

Fever and weight loss were common complaints. Fever was present in $70 \%$ patients in the series. Three

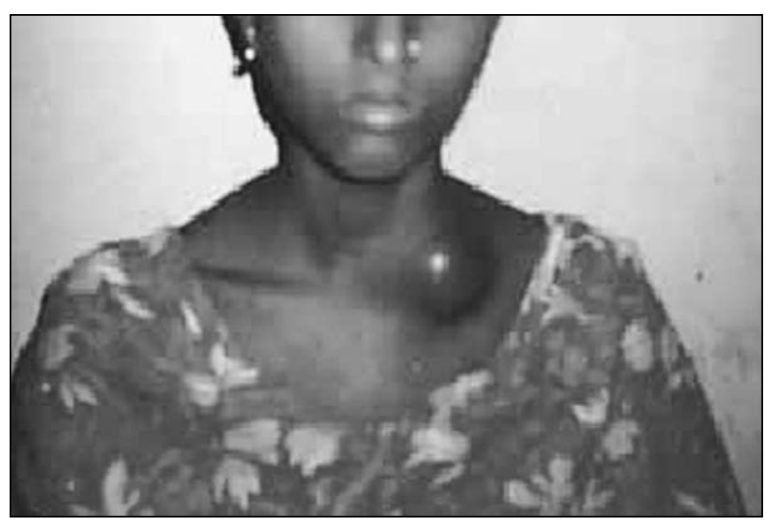

Fig.-1: Cold abscess before burst fourths of them had low-grade evening rise of temperature. Only a quarter of the patients had high fever in the course of illness. Weight loss was seen more frequently $(81.2 \%)$.

Though cough was complained by only $30 \%$ of patient half of them had productive cough. 45\% patients were clinically anemic.

Twenty-nine patients of this series had tubercular lymphadenopathy including secondary cold abscess (Fig.-1,2). Nearly half of them had involvement of multiple groups. One third had only cervical lymphadenopathy. Posterior triangle group being more commonly affected. One had submandibular lymph node involvement. This is shown in Table-IV. Table-V shows physical examination findings of different lymph node groups.

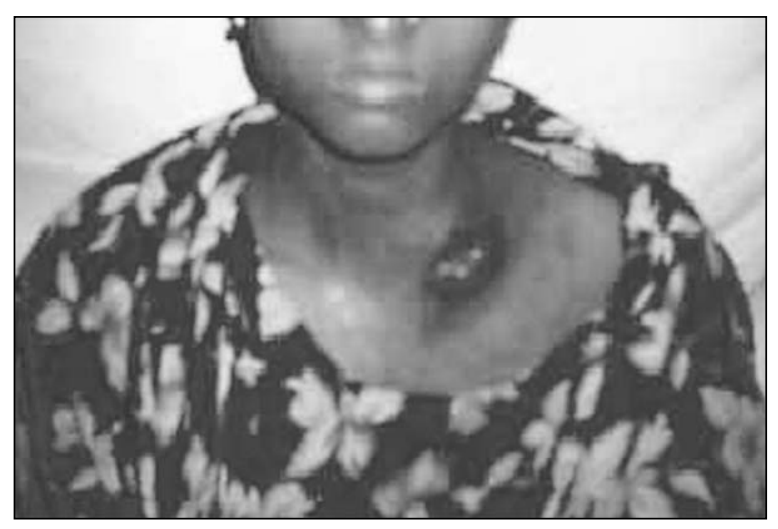

Fig.-2: Cold abscess after burst

Table-IV

\begin{tabular}{|c|c|c|c|c|c|}
\hline \multicolumn{6}{|c|}{ Distribution among the different groups of lymph nodes $(n=29)$} \\
\hline \multirow[t]{3}{*}{ Cervical } & Side & Superficial & Deep & Posterior & Spraclavicular \\
\hline & Right & 4 & 8 & 13 & 8 \\
\hline & Left & 2 & 5 & 14 & 5 \\
\hline \multirow[t]{3}{*}{ Axillary } & Side & Anterior group & Posterior & Central & Apical \\
\hline & Right & 5 & 1 & 10 & 0 \\
\hline & Left & 5 & 2 & 11 & 2 \\
\hline \multirow[t]{3}{*}{ Inguinal } & Side & Superficial & \multicolumn{2}{|c|}{ Deep } & \\
\hline & Right & 7 & \multicolumn{2}{|c|}{5} & \\
\hline & Left & 6 & \multicolumn{2}{|c|}{5} & \\
\hline \multirow[t]{3}{*}{ Submandibular } & Side & Single & \multicolumn{2}{|c|}{ Multiple } & \\
\hline & Right & 1 & \multicolumn{2}{|c|}{4} & \\
\hline & Left & 0 & \multicolumn{2}{|c|}{5} & \\
\hline
\end{tabular}


Table-V

\begin{tabular}{lcccccccc}
\multicolumn{2}{c}{$\begin{array}{c}\text { Presentation and findings of lymph } \\
\text { Features }\end{array}$} & Cervical group & Axillary group & Inguinal group & \multicolumn{2}{c}{$\begin{array}{c}\text { Submandibular } \\
\text { group }\end{array}$} \\
& Right & Left & Right & Left & Right & Left & Right & Left \\
\hline Single Node & 4 & 5 & 6 & 6 & 0 & 0 & 2 & 1 \\
Multiple Nodes & 17 & 15 & 6 & 7 & 6 & 7 & 3 & 3 \\
Discrete & 6 & 10 & 8 & 8 & 3 & 7 & 2 & 2 \\
Matted & 11 & 8 & 4 & 3 & 1 & 0 & 1 & 2 \\
Rubbery & 1 & 3 & 2 & 3 & 1 & 2 & 1 & 1 \\
Pain & 7 & 11 & 4 & 2 & 1 & 0 & 2 & 2 \\
Tenderness & 8 & 11 & 4 & 2 & 1 & 0 & 2 & 1 \\
Irregularity & 3 & 5 & 1 & 1 & 1 & 0 & 0 & 0 \\
Fixity with overlying skin & 9 & 10 & 1 & 1 & 0 & 0 & 2 & 1 \\
Fixity with underlying structures & 14 & 13 & 4 & 3 & 2 & 2 & 4 & 3 \\
Abscess & 6 & 5 & 0 & 0 & 0 & 0 & 0 & 0 \\
Discharging sinus & 4 & 1 & 2 & 2 & 0 & 0 & 0 & 0 \\
\hline
\end{tabular}

Twenty-eight cases (35\%) had operations for abdominal tuberculosis. Of these half had involvement of small gut only in the form of stricture and stenosis. 10 (35\% of abdominal tuberculosis) cases had lump in the right iliac fossa due to involvement of ileo-caecal region. Mesenteric lymph nodes were involved in all cases (Table-VI)
Presentation in majority of cases were chronic abdominal pain, lump in right iliac fossa or signs of intestinal obstruction. One-third patients were admitted with acute complications like perforation or obstruction. One of them had clinical diagnosis of acute appendicitis. Clinical findings are presented in Table-VII.

Table-VI

\section{Distribution of sites of abdominal tuberculosis along with percentage $(n=28)$}

\begin{tabular}{lcc} 
Sites & Number of cases & Percentage \\
\hline Small gut & 14 & 50 \\
Ileo-caecal & 10 & 35.7 \\
All parts & 4 & 14.3 \\
Lymph nodes & In all cases & 100 \\
\hline
\end{tabular}

Table-VII

\begin{tabular}{|c|c|c|c|c|c|}
\hline Acute Abdomen & Cases & Percentage & Chronic Abdomen & Cases & Percentage \\
\hline$\overline{\text { Pain }}$ & 10 & 100 & Pain & 11 & 61.1 \\
\hline Perforation & 7 & 70 & Lump & 10 & 55.5 \\
\hline Peritonitis & 7 & 70 & Obstruction & 8 & 44.4 \\
\hline Obstruction & 3 & 30 & Visible peristalsis & 7 & 38.9 \\
\hline Shock & 2 & 20 & Ascites & 4 & 22.2 \\
\hline Intussusception & 1 & 10 & Alteration of bowel habit & 4 & 22.2 \\
\hline Appendicitis & 1 & 10 & & & \\
\hline
\end{tabular}


Nine cases of tubercular abscess were found in different parts of the body, three cases in the back of chest, three cases in the back, neck abscess were found in two cases and perianal abscess in two.

Five cases of breast abscess were found. Two of them were pregnant. They presented with pain, lump, abscess and discharging sinus in the breast. In two cases there were axillary nodal involvement. In one case there were abscess in right thigh (Fig.-3).

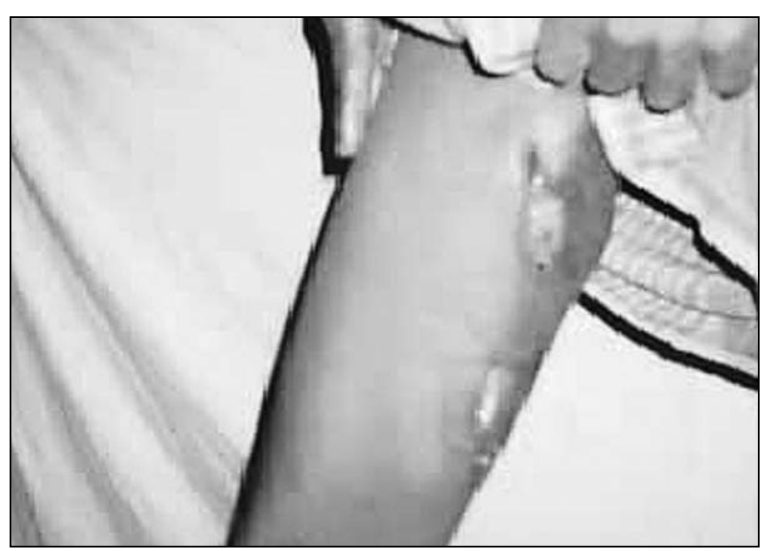

Fig.-3: Right thigh abscess (after drainage)

Diagnosis was based on histopathological findings of caseating or non caseating granuloma (Fig.-4). Excision and biopsy were done in 41 cases (51.2\%) and lymph node biopsy in 26 cases (32.5\%). Only in eleven cases FNAC were done from lymph node or the palpable lump and tuberculosis was confirmed. Two patients with abscess had AFB on stain (Fig.-5). Spinal tuberculosis was diagnosed on the basis of clinical and typical radiological findings (supplemented by FNAC).

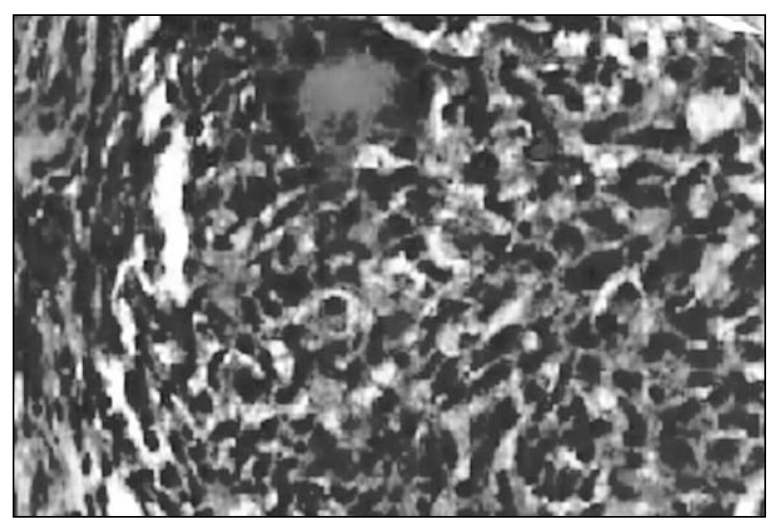

Fig.-4: Tuberculous granuloma

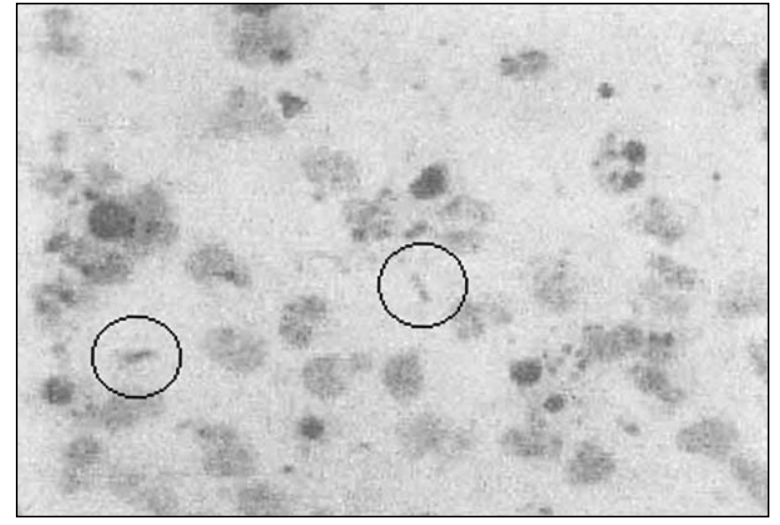

Fig.-5: Mycobacterium tuberculosis in AFB stain

$22.3 \%$ patients $(\mathrm{n}=16)$ had $\mathrm{Hb} \%$ below $10 \mathrm{gm} / \mathrm{dl}$, $70.5 \%$ patients $(n=50)$ had ESR above $50 \mathrm{~mm}$ in $1^{\text {st }}$ hour, $26.5 \%$ patients $(n=18)$ had features of pulmonary tuberculosis in chest X-ray, 66.6\% patients $(n=34)$ had positive tuberculin test.

Surgical interventions were needed in almost all patients either for diagnosis or for therapeutic purpose. It is shown in Table-VIII. Lymph node biopsy was done in 26 patients $(32.5 \%) .10$ patients $(12.5 \%)$ were treated by incision and drainage for their abscesses including breast. Daily dressing was done later followed by secondary closure. Resection and anastomoses were required in $13.7 \%$ patients $(n=$ 11) for stricture, stenosis and perforation in the ileum and jejunum. Stricturoplasty was done in one patient. Ileostomy was done in two patients for diffuse peritonitis. Right hemicolectomy was done in $10 \%$ $(n=8)$ patients for lesion in the terminal ileum and ileo-caecal growth. Perforation of ileum was repaired in two cases. One had leakage and ileostomy was done later on. Aseptic aspiration of pus was done in two cases for sternal abscess. Later both these were referred to Institute of Chest Disease and Hospital for further management. Laminectomy with removal of extradural mass was done in one case. Right-sided orchidectomy was done in one patient with testicular tuberculosis.

All patient were treated with standard anti-tubercular chemotherapy except one who died in early postoperative period.

Forty-eight patients came for follow up out of which 44 patients responded completely to anti-tubercular chemotherapy. Adverse effects in the form of 
jaundice and optic neuropathy were noted in two cases and treated successfully. There was no response to drugs in 4 cases. Culture and sensitivity for Mycobacterium tuberculosis were done and treated accordingly. Relapse were noted in two cases and were treated with second line therapy with addition of Ciprofloxacin $(750 \mathrm{mg})$ twice daily for initial two months in one of them and co-amoxiclav $(625 \mathrm{mg})$ thrice daily for initial two months in other patient along with conventional antituberculous therapy. Both responded with the above medication.

\section{Discussion:}

The primary objective of this study was to record clinical presentation in confirmed cases of extrapulmonary tuberculosis. The study showed neck swelling as the commonest presenting feature $(31.2 \%)$, which indirectly represents tubercular cervical lymphadenopathy. Including other groups, lymph node tuberculosis is $36.2 \%$, which is the commonest of extra-pulmonary tuberculosis in this series. Similar observations were also reported by Faiz in $1990^{6}$, Alvarez $1984^{7}$, Farer $1979^{8}$, Mokhtar $1983^{9}$ and Froude et al, $1982^{10}$. The patients presented with abdominal pain and distension were $22.5 \%$ and $17.5 \%$ respectively. These were the patients of abdominal tuberculosis, which is $35 \%$ in this study. It is similar to data of Medical Research Council. Surveyed in 1983 for Indian subcontinent (ISC) ethnic group in which lymph node tuberculosis was $52 \%$ followed by abdominal tuberculosis $(14 \%)^{11}$.

Majority of the patients $n=32(40 \%)$ were between 21-30 years age group followed by $31.2 \%$ in $12-20$ years age groups. So $71.2 \%$ of patients are under 30 years of age, which is similar to observation of Hayati-IN et al ${ }^{12}$.

There is overall preponderance of female (56.3\%) as compared to male (43.7\%). But Faiz in his study showed $62 \%$ were male and $38 \%$ were female ${ }^{6}$. Farer et al, reported 55\% male and $45 \%$ female ${ }^{8}$.

Incidence of extra-pulmonary tuberculosis is more common among the housewives (37.5\%), which is $48 \%$ in another study conducted by Chowdhury in Chittagong Medical College Hospital on lymph node tuberculosis ${ }^{13}$. But starting from university teacher to prisoner all were affected, though lower class were frequently $(66.2 \%)$ affected. Among the affected patients $56 \%$ were vaccinated against tuberculosis. So, vaccinated patient may also be affected by tuberculosis. This observation is similar to the study done earlier by Chowdhury ${ }^{13}$. In our country housewives or female family members are responsible for taking care of ill patients. This may be the cause of female preponderance of housewives in this study.

About $22.5 \%$ of patients were smoker. Similar proportions have the history of intake of unpasteurized milk. So, milk still may be transmitting media of tuberculosis. This was also observed by Islam and Quayum ${ }^{14}$. 36.2\% patients had the history of contact with a diagnosed case of pulmonary tuberculosis, which is $38 \%$ in the study made by Chowdhury ${ }^{13}$.

In this study population, $18.8 \%$ patients had previous history of tuberculosis of which $73.3 \%$ were incompletely treated. Being a curable disease it needs much public awareness about the outcome of tuberculosis both from medical practitioner and government sector. $23.8 \%$ patients had family history of tuberculosis of which most were treated and cured $(72 \%)$ with a few maltreated (11.1\%) and untreated $(5.5 \%)$ cases. Being a communicable disease other family members of a tuberculous patient should be aware of it and must take necessary measures to avoid the transmission of the disease.

Forty-two (52.5\%) patients came from overcrowded condition. In this study, $93.7 \%$ of patients came from lower and middle class. Same observations were noted by Chowdhury ${ }^{13}$. So, this confirms that tuberculosis is more common among the lower socioeconomics. None was on immunosuppressive therapy in the study population.

In this study 56 patients $(70 \%)$ had the history of fever - $68 \%$ had low grade, $71.4 \%$ having evening peak and in $62.5 \%$ of cases subsides with sweating. $30 \%$ patients had no history of fever at all. $30 \%$ patients had history of cough of which $15 \%$ were unproductive and 15\% were productive.

Among the study population $45 \%$ of patients had various degree of anemia, 68 patients (85\%) had 
significant weight loss. Significant weight loss was also noted among the higher socio-economic class. So, weight loss is the single most common physical abnormality among the extra-pulmonary tuberculosis. Weight loss was also documented in other studies Chowdhury ${ }^{13} 81 \%$.

22.3\% patients had $\mathrm{Hb} \%$ below 10 gm/dl, ESR was more than $50 \mathrm{~mm}$ in $1^{\text {st }}$ hour in $70.5 \%$ patients. $26.5 \%$ patients had pulmonary lesion on chest X-ray. Tuberculin test was positive in $66.6 \%$ of patients. Similar observation were found by Lau-SK et al $^{15,16,17}$, Chowdhury ${ }^{13}$.

In this study lymph node tuberculosis was found to be common (36.2\%) among the extra-pulmonary tuberculosis, cervical group was most commonly affected. Cervical with other groups of involvement were $48.2 \%$. It correlates with study conducted by Chowdhury ${ }^{13}$ in very recent past. Posterior group of cervical lymph node were commonly involved. Multiple nodes even in a single group were frequently involved. Lymph node tuberculosis in USA is 30\%, in England $50 \%$ and in Hong Kong $45 \%$ of total extrapulmonary tuberculosis ${ }^{1,18}$.

Among the abdominal tuberculosis small gut was frequently affected (50\%) followed by ileo-caecal tuberculosis (35.7\%). All patients were presented with abdominal pain in acute cases and $61 \%$ in chronic cases. Hossain ${ }^{19}$ also observed pain (45\%) as the commonest presenting feature followed by right iliac fossa lump (42\%). Among the chronic cases $55.5 \%$ patients presented with right iliac fossa lump. Few cases presented with intestinal perforation, obstruction and peritonitis. Plain X-ray of abdomen was done in acute cases and barium follow through in chronic cases. Sometime patient presented with leaky intestinal perforation having no free gas shadow.

Nine cases $(11.2 \%)$ presented with abscess in different parts of the body. All were drained followed by chemotherapy. In this study five cases $(6.2 \%)$ were breast TB including two pregnant women, who were also treated successfully. A literature review by Morgan in $1931^{20}$ revealed 439 cases of tuberculous mastitis with the incidence between 0.5 and 1.04 per cent. Of approximately 8000 breast specimen studied, Haagensen $^{21}$ reported only five cases of breast TB between 1938 and 1967. Only 500 cases were documented from the world literature by Hamit and Rangsdale in $1982^{22}$. Less than 100 cases of breast TB were reported from India till $1987^{23}$. Several Indian series reported the incidence of breast TB amongst the total number of mammary conditions to vary between 0.64 and 3.59 per cent ${ }^{24,25}$. Diagnosis of breast TB specially in pregnancy is problematic owing to enlargement of breast, manifestations are masked. The disease is often overlooked and misdiagnosed as carcinoma or pyogenic abscess ${ }^{26}$. Opinion varies for diagnostic aids between FNAC and incision biopsy. FNAC is sometimes inconclusive. Occasionally tissue taken by incision biopsy cannot give the real reports suggestive of tuberculosis. Delay in wound healing occurred in pregnant women's breast. Breast TB caused by an atypical mycobacterium has recently been reported by Verfaillie $\mathrm{G}$, et $\mathrm{al}^{27}$.

Diagnosis was based on either histopathological or cytopathological (FNAC) examination (97.5\%) suggestive of tuberculosis or demonstration of AFB (2.5\%). Though 87 patients were studied but only 80 patients were included in this study. Excluded group were clinically consistent with tuberculosis, treated with anti-tuberculous chemotherapy but there was no specific histological or cytological evidence to prove tuberculosis.

Lymph node biopsy is the commonest (32.5\%) surgery performed followed by resection and anastomosis $(13.7 \%)$. Incision and drainage were done in $12.5 \%$ and limited right hemicolectomy in $10 \%$ of patients. Most of the patients were afraid of surgery even a small procedure like lymph node biopsy. Medical treatments were provided in all patients. Most of the patients were unable to buy such a costly drugs for a long duration of 6, 9, 12 months or so. Sometimes they have false belief in such a long duration of therapy. Irregular intake of chemotherapy was frequently encountered. The patients were anxious about the adverse effect of multiple drugs. Most of them complained of their intolerability of drugs in spite of having no such harmful effects at all. Free drugs were supplied to the patient irregularly from hospital and upazilla health complex owing to non-availability of drugs during the study period. Sometime patient discontinued the drugs due to this reason. 
$37.5 \%$ patients among the follow up group responded well to anti-tubercular chemotherapy. In three cases lymph nodes were enlarged further during the course of chemotherapy but subsided later on. One axillary lymph node in a female persisted after one year of treatment. Excision biopsy of it showed non-specific lymphadenitis. Few patients developed adverse effect and they were treated accordingly. Culture and sensitivity was done in non-responder and drugs were changed according to culture and sensitivity report. But long time required for culture and sensitivity report is a problem. In the mean time few patients were lost from the follow up.

Two patients (prisoner) having sternal swelling (abscess) with referred to the Institute of Chest Disease and Hospital in Dhaka.

Majority of poor patients did not come for follow up. Poor socio-economic condition, social, familial bindings and illiteracy may be responsible.

This study does not reflect the prevalence of extrapulmonary $\mathrm{TB}$ in our country but indicates the diversity of clinical presentation and the diagnostic problem that might be encountered even in a country where tuberculosis is still common. Diagnosis requires high clinical suspicion, special diagnostic procedures, special staining, and culture media for acid fast bacilli. Clinical diagnosis combined with strongly positive tuberculin test, high ESR with significant weight loss may suggest tuberculosis. Delayed diagnosis results in increasing morbidity, mortality and cost to the health care system. Particularly in areas of high endemicity of Mycobacterium tuberculosis like us clinician should be aware of the various forms of extra pulmonary tuberculosis. Early diagnosis will prevent mortality and morbidity from this dreadful but curable disease. The available epidemiology in Bangladesh is presented in this study, but requires further study in a large scale.

\section{Acknowledgement}

With a deep sense of gratitude we remember all of our patients who in their great pain and distress helped in collection the clinical data and information for this study.
We are grateful to the Director of $\mathrm{CMCH}$ for permission to conduct this study and to all colleagues of Dept. of surgery, pathology, histopathology, microbiology and radiology for helping a lot by supplying data and report related to this study.

\section{References:}

1. Medical Research Council. National Survey of Tuberculosis Notifications in England and Wales in 1983: characteristics of disease. Tubercle, 1987; 68: 19-32.

2. Medical Research Council Cardiothoracic Epidemiology Group. National survey of notifications of tuberculosis in England and Wales in 1988. Thorax, 1992; 47: 770-5.

3. Weir, M. R. and Thornton, G. F. Extra Pulmonary Tuberculosis. Am. J. Med. 1985; 79: 467-78.

4. Pitchenik AE, Fertel D, Bloch AB. Pulmonary effects of AIDS: Mycobacterial disease-epidemiology, diagnosis, treatment, and prevention. Clin. Chest Med. 1988; 9: 425-41.

5. Wolinsky E. Tuberculosis, Wyngaarden JB, Smith LH editors. In Cecil Textbook of Medicine, 18th edn. W. B. Saunders, Philadelphia 1988; 2: 1682-92.

6. Faiz MA. Extrapulmonary tuberculosis in Bangladesh -A review of 47 case. J Bangladesh Coll Phys and Surgeon 1990; 7: 1-7.

7. Alvarez $\mathrm{S}$ and McCabe WR. Extrapulmonary tuberculosis revisited: A review of experience at Boston city and other hospitals. Medicine, 1984; 1: 25-55.

8. Farer LS, Lowel AM and Meador MP. Extrapulmonary tuberculosis in the United States. AM J Epidemiol, 1979; 109: 205-217.

9. Moktar A and Salman K. Extrapulmonary tuberculosis. Saudi Med. J 1983; 4: 317.

10. Froude JR and Land Kingston M. Extrapulmonary tuberculosis in Saudi Arabia. A review of 162 cases. King Faisal Specialist Hosp. Med. J. 1982; 2: 85.

11. Davies, P.D.O. Clinical tuberculosis. First Edition 1994, Reprint 1995.

12. Hayati-In, Ismail-Y Zarkarmain-Y EPT; a two year review of cases at the General Hospital, Kota Bharee Med J Malaysia, 1993, 48; 416-20.

13. Chowdhury M.I. A clinical study on lymph node tuberculosis- dissertation of BCPS 2003.

14. Islam MN, Quayum MA. Abdominal tuberculosis- A review. Northern Med. J. 2001: 10: 85-89. 
15. Lau, S. K. Wei, W.I. Hsu, C. and Engzell, U.C. Fine needle aspiration biopsy of tuberculous cervical lymphadenopathy. Aust. NZ J. Surg., 1988; 58: 947-50.

16. Lau, S. K. Wei. W. I. Kwan, S. and Yew, W. W. Combined use of fine-needle aspiration cytologic examination and tuberculin skin test in the diagnosis of cervical tuberculous lymphadenitis: a prospective study. Arch. Otolaryngol. Head Neck Sug., 1991; 117: 87-90.

17. Lau, S. K. Wei, W. I. Hsu, C. and Eagzell. U. C(1990) Efficacy of fine needle aspiration cytology in the diagnosis of tuberculous cervical lymphadenopathy. J. Laryngol-Otol., 1990; 104: 24-7.

18. Dandapat, M. C., Mishra, B. M. Dash, S. P. and Kar, P. K. (1990) Peripheral lymph node tuberculosis: a review of 80 cases. Br. J. Surg., 1990; 771: 911-2.

19. Hussain G.M.Z. Gastro-intestinal tuberculosis- study of 62 cases. BJMS 2001. 7, 2, 75-8.

20. Morgan M. Tuberculosis of the breast. Surg Gynecol Obstet 1931; 53: 593-605.
21. Haagensen CD Infections in the breast. Haagensen CD.(ed). In: Disease of the breast.3rd ed. Philadelphia: WB Saunders; 1986 p.384-93.

22. Hamit HF, Ragsdale TH,Mammary tuberculosis. J R Soc Med 1982; 75: 764-5.

23. Banerjee SN, Ananthakrishnan N, mehta RB, Prakash S, tuberculous mastitis: a continuing problem. World J Surg 1987; 11: 105-9.

24. Dharkar RS, Kanhere MH, Vaishya ND, Baisarya AK. Tuberculosis of the breast. J Indian Med Assos 1968; 50: 207-9.

25. Mukerjee P, George M, Maheshwari HB, Rao CP. Tuberculosis of the breast. J Indian Med Assos 1974; 62: 410-2.

26. Green RM, Ormerod LP, Mammary tuberculosis: rare but still present in the United Kingdom. Int J tuberc Lung Dis 2000; 4: $788-90$

27. Verfaillie G, Goossens A, Lamote J, Atypical mycobacterium breast infection. Breast J 2004; 10: 60 . 